\title{
What do we really know about
}

\section{infants who attend Accident and}

\section{Emergency departments?}

\section{Authors}

\section{Michelle Heys}

UCL Institute for Global Health, 30 Guilford Street, London, WC1N 1EH, UK Child Public Health Group, Imperial College River Island Academic Department, Paediatric Department, Northwick Park Hospital (NWLH NHS Trust), London, UK

Email:m.heys@ucla.co.uk

\section{Ho-Ming Kwong}

Imperial College London,

Medical School London, UK

\section{Jo Reed}

Child Public Health Group, Imperial College River Island Academic Department, Paediatric Department, Northwick Park Hospital (NWLH NHS Trust), London, UK

\section{Mitch Blair}

Child Public Health Group, Imperial College River Island Academic Department, Paediatric Department, Northwick Park Hospital (NWLH NHS Trust), London, UK; Imperial College London, London, UK

\section{Corresponding author:} Michelle Heys, as above

\section{Keywords}

infant; accident and emergency; emergency service; hospital; clinical audit; diagnosis; clinical coding

\section{Abstract}

Aims: Accident and Emergency attendances continue to rise. Infants are disproportionately represented. This study examines the clinical reasons infants attend UK Accident and Emergency departments.

Methods: A retrospective review of 6,667 infants aged less than one year attending Accident and Emergency at two district general hospitals in London from $1^{\text {st }}$ April 2009 to $30^{\text {th }}$ March 2010. All infants had been assigned to a diagnostic category by the medical coding department according to National Health Service (NHS) data guidelines, based on the clinical diagnoses stated in the medical records. The Accident and Emergency case notes of a random subsample of $10 \%$ of infants in each of the top five recorded diagnostic categories $(n=535)$ were reviewed in detail and audited against the standard national NHS data set.

Results: The top 5 clinical diagnoses were 'infectious diseases', 'gastrointestinal', 'respiratory', 'unclassifiable' and 'no abnormality detected' (NAD). A third of infants were originally given a diagnosis of unclassifiable (21.5\%) or NAD (11.5\%). After detailed case-note review, we were able to reduce this to $9.7 \%$ (95\% confidence interval $(\mathrm{Cl}): 9.0,10.4)$ and 8.8\% (95\% Cl: 8.1, 9.5), respectively.

Conclusion: This study demonstrates the importance of providing a clear clinical diagnosis and coding system for Accident and Emergency attendances and understanding that system fully. This would allow for better informed health service evaluation, planning and research as each of these relies on the interpretation of routine health-care data. Furthermore, the relatively high proportion (10\%) of infants attending with no discernible underlying medical abnormality suggests the health needs of a significant proportion of infants attending Accident and Emergency departments may be better addressed by alternative service provision and/or improved education and support to parents.

\section{BACKGROUND}

Across UK Accident and Emergency (A\&E) departments, attendance rates are rising..$^{1,2}$ There were 908 attendances per 1,000 infants aged less than one year at London A\&Es in the financial year 2010-2011. ${ }^{3}$ Babies, young children and adults over 65 years are at particular risk of unplanned hospital admission. ${ }^{4}$ Short-stay admissions for minor illness in children have risen, the majority of which present through A\&E. ${ }^{5}$ Families often lack the traditional support of extended families, and there has been a substantial decrease in numbers of health visitors in the past five years who can support parents requiring expert advice. Infants aged less than one year are a highly vulnerable group attending A\&E whose health-care needs may be better met by alternative health service provision. ${ }^{6}$

The structure of the UK National Health Service (NHS) is changing rapidly. The acute hospital care spend for children and young people accounts for 50\%-60\% of allocated clinical commissioning group (CCG) budgets (as seen in unpublished data, M Blair, 2013). There is a growing focus on improving the primary secondary care interface, ${ }^{7}$ to help reduce unnecessary use of acute care through improved primary care provision, ${ }^{8}$ patient education ${ }^{9}$ and 
clearer signposting to appropriate local services. ${ }^{10,11}$

Detailed information regarding clinical reasons for presentation to $A \& E$ is lacking. ${ }^{11,12}$ This study aims to describe, review and revise the clinical reasons for attendance to A\&E for infants aged less than one year.

\section{METHODS}

\section{Study design}

A retrospective review of routine hospital activity data of 6,667 infants and an in-depth case-note audit of 535 infants aged less than one year attending A\&E at two large, district general hospitals (DGHs) in London over the period of $1^{\text {st }}$ April 2009 to 30th March 2010 was carried out.

Ethics approval was sought from the ethics committee of the NHS Trust covering both DGHs; however, as this is an audit and a service evaluation, they concluded that the study did not require a formal ethics review.

\section{Setting}

The setting comprised of two large DGHs which are part of a single NHS Hospital Trust in London (serving a population of around 500,000 people in total), with different models of A\&E care; Hospital A was paediatric-led with all children and young people aged less than 16 years being seen by a paediatrician, and Hospital B was predominantly A\&E-led with infants aged less than one year and all potential admissions in children and young people aged less than 16 years being seen by a paediatrician.

\section{Data}

All data were anonymized by a single researcher. Routinely collected hospital data were reviewed for all 6,667 of the infants. Data were available on demographic, clinical and process characteristics. Demographic information included age, sex, postcode, ethnicity, distance lived from the hospital and socio-economic position (SEP). Age was calculated in months from the date of birth to date of admission. Ethnicity was recorded as stated by the accompanying adult on attendance and revised from 18 into 5 categories (as per Table 1). Postcode was recorded on admission and was used to derive both distance lived from the hospital and an index of SEP. The distance lived from hospital to home was calculated using the UK Department for Education online tool, which calculates the straight line distance between two postcodes. ${ }^{13}$

The measure of SEP used was the Income Deprivation Affecting Children Index (IDACI), and was derived from the postcode where stated using the UK Department for Education online tool. ${ }^{14}$ The IDACl gives both a rank and score for the super output area (SOA) within which the postcode lies. The IDACI score ranges from 0 to 1 , with 0 being the lowest deprivation and 1 being the highest, such that a score of 0.24 would equate to $24 \%$ of children less than 16 years in that area living in families that are income deprived, which is defined as being in receipt of income support and an equivalized income which, excluding housing benefits and before housing costs, is less than $60 \%$ of the national medium income. This 60\% threshold depends on household composition, and the latest figures from 2008/2009 state the weekly threshold as follows: single adult, no children - £119 (US\$182); couple with no children - £206 (US\$315); single adult with two dependent children - £202 (US\$309); and couple with two dependent children under 14 years of age £288 (US\$440). The IDACl also ranks each SOA within the United Kingdom from most to least deprived with a range of 0-32,482.

Clinical characteristics included presenting complaint, final diagnosis, admission outcome and number of $A \& E$ attendances. On arrival in A\&E, the administration staff recorded the presenting complaint given by the adult attending with each infant. This was entered as free text into the hospital activity database. For those infants whose case notes were reviewed, we also recorded the presenting complaint as written in the medical clerking notes. Whether the infant was admitted (to any hospital) or not was derived from the hospital database. The number of A\&E attendances during the study period was calculated for each infant.

Process data included hospital, source of referral to A\&E and date and time of admission. Hospital site was coded as either Hospital A or Hospital B. The source of referral was recorded in the hospital database for all infants as 'general practitioner' (GP), 'self-referral', ' health-care provider', 'other' or 'police'. Time of attendance was categorized into three time periods: 9 a.m. to 4.59 p.m., 5 p.m. to 9.59 p.m. and 10 p.m. to 8.59 a.m. Season of admission and day of week of admission were derived from the date of admission.

\section{Outcome - final diagnosis}

The main outcome of interest was final diagnosis, first as recorded in the hospital activity database and second as revised by the research team. Medical coding staff trained in diagnostic coding are employed in each hospital to review case notes and enter a diagnosis and a diagnostic category to the hospital activity database. The diagnoses and diagnostic categories are taken from the NHS Data Dictionary - a standard which is used in all hospitals. ${ }^{15} \mathrm{~A}$ diagnosis of 'unclassifiable' is given when the coding staff are unable to interpret a diagnosis from the medical notes or when the diagnosis is one of those listed under that category (see Table 2). For example, a diagnosis of a tumour (benign or otherwise), fever or parental concern would all be categorized under unclassifiable diagnosis. A diagnosis of no abnormality detected (NAD) would have been given when the clerking notes contained a written diagnosis of 'NAD'.

In Hospital A, all A\&E attendances were clerked using a structured standard proforma which included a box for diagnosis at the end of the proforma. In Hospital B, all A\&E attendances were clerked using free text on standard note paper with a box for diagnosis on the covering sheet. The medical experience of the assessing paediatric doctor will have varied.

In all, $80 \%$ of infants were assigned one of five diagnostic categories: 
Table 1

Demographic, clinical and process characteristics of all infants in the top five diagnostic categories $(n=5,080)$ compared with the subsample $(n=535)$

\section{Characteristic}

Male gender

Age (months), mean (SD)

Ethnicitya

Distance lived from hospital (km), mean (SD)

Distance lived from hospital in quartiles

\begin{tabular}{|l|l|}
\hline & Second \\
\hline & Third \\
\hline IDACl-quartile & Fourth (furthest distance) \\
\hline Furst (least deprived) \\
\hline $\begin{array}{l}\text { Number of A\&E attendances, } \\
\text { mean (SD) }\end{array}$ & Second \\
\hline Hospital A & Third \\
\hline Source of referral & Fourth (most deprived) \\
\hline & \\
\hline & Golice \\
\hline & Self-referral \\
\hline
\end{tabular}

All infants in top five diagnostic categories 55.9

$$
5.1(3.7)
$$

31.8

19.3

3.7

Mixed background

Not stated or other

White

18.0

27.3

$5.0(16.5)$

24.8

24.8

25.5

25.0

25.0

24.4

25.6

24.9

24.6

1.7 (1.3)

43.4

11.8

0.3

19.3

1.4

67.1

\section{Subsample}

63.0

$5.2(3.7)$

0.006

0.806

32.7

18.1

2.8

0.786

18.3

28.0

$$
4.3(10.7)
$$

0.364

25.5

26.9

22.6

25.1

23.9

25.8

22.2

0.193

28.1

21.5

0.116

$1.6(1.1)$

0.090

45.8

0.505

9.4

0.0

20.4

0.273

1.1

69.2

.006

806

0.786

0.364

p-value 
Table 1 (continued)

\section{Characteristic}

Time period of attendance

Season of attendance

Day of week of admission

infectious disease (ID), gastrointestina tract (GIT), respiratory, unclassifiable and NAD. A random sample of $10 \%$ of infants in each of these five diagnostic categories was created using an online random number generator. ${ }^{16}$ The representativeness of this subsample was assessed using chi-square analysis (categorical) and analysis of variance (ANOVA; continuous) for each of the descriptive variables. The A\&E case notes of these 535 infants were then reviewed in detail by both junior and senior authors. Uncertainty in the coding process was discussed with the head of coding for A\&E for the trust. For each of the 535 infants, the interpreted diagnosis was recorded based on what was clearly recorded in the medical notes and recoded these revisions according to the NHS Data Dictionary. Where no diagnosis was recorded in the medical notes, a diagnosis of unclassifiable was given as before. Finally, an estimate of estimated the revised proportion of infants that would have been in each of the top five diagnostic categories was made assuming that the subsample was representative of the whole sample. Data analysis was done using STATA v. 12 (STATA Corporation, College Station, TX).

\section{RESULTS}

Of the 44,500 children aged 16 years and under who attended A\&E across both hospitals during the study period, 6,667 (13.5\%) were infants aged less than one year - representing the single largest age category (by year) for all children and young people attending A\&E. The mean age was 5.3 months (standard deviation (SD) $=3.8$ months) and more male (56.2\%) than female infants attended A\&E.

Table 1 shows the demographic, clinical (excluding presenting complaint) and process characteristics for the subsample compared with the total sample of infants who received the top five clinical diagnoses of 'ID', 'GIT', 'respiratory', 'unclassifiable' and 'NAD' $(n=5,359$, 80.4\%). By chance, slightly more male than female infants were randomized to the subsample. Otherwise, the subsample was similar in every other demographic, clinical and process characteristic. 


\section{Percentages of children assigned to each of the top five diagnostic categories: original and revised coding}

\begin{tabular}{|l|c|c|c|}
\hline $\begin{array}{l}\text { Diagnostic } \\
\text { category }\end{array}$ & $\begin{array}{c}\text { \% infants } \\
\text { original coding }\end{array}$ & \multicolumn{2}{|c|}{$\begin{array}{c}\text { Estimated \% infants } \\
\text { revised coding (95\% Cl) }\end{array}$} \\
\hline ID & 22.2 & 24.2 & $(23.2,25.2)$ \\
\hline Unclassifiable & 21.5 & 9.7 & $(9.0,10.4)$ \\
\hline GIT & 15.9 & 18.8 & $(17.9,19.8)$ \\
\hline NAD & 11.5 & 8.8 & $(8.1,9.5)$ \\
\hline Respiratory & 9.4 & 12.0 & $(11.2,12.8)$ \\
\hline Other & 19.6 & 26.7 & $(25.7,27.8)$ \\
\hline
\end{tabular}

Cl: Confidence intervals derived using Wilson procedure without correction for continuity; ${ }^{21,22}$ ID: infectious disease; GIT: gastrointestinal tract (including gastroenteritis); NAD: no abnormality detected; NHS: National Health Service.

ID category includes the following: paediatric petechial rash, chickenpox, herpes zoster: shingles, influenza, meningitis, measles, malaria, mumps, rubella, typhoid and sexually transmitted disease. Respiratory category includes the following diagnoses relevant to paediatrics: pleural effusion, paediatric croup/stridor, paediatric respiratory distress, paediatric viral induced wheeze, cough, cause unspecified, haemoptysis, pulmonary embolism, pneumothorax, pleurisy, pleural effusion, respiratory arrest, neonatal respiratory distress, haemothorax and haemopneumothorax.

Unclassifiable: defined as unclassifiable if no diagnosis given clearly in the medical notes or if the diagnosis was included in the NHS database for diagnostic codes as unclassifiable, which includes the following diagnoses: pain/neuralgia, paraesthesia: cause unknown, degenerative problem, benign tumour, non-benign tumour, congenital problem, organ failure, not specified problem, paediatric pyrexia, paediatric parental concern, unwell child (see free text), arthritis, cramps, costochondritis, elderly: immobile (off legs); dehydration, electrolyte disturbance, gout, hypothermia, hiccups, lymphadenopathy, musculoskeletal pain, oedema, osteoporosis, parasitic infestation, pyrexia, sarcoidosis, test review or repeat, vertigo: unspecified cause, wasting disease, cachexia, neonatal congenital abnormality, neonatal metabolic disease, removal of sutures, bursitis, baker's cyst, cyst, carpal tunnel syndrome, dysphagia, epistaxis mass: unspecified, osteoarthritis, prolapse, post-operative complication, parotid problem, paraphimosis, rupture, stone, torsion, tube/ostomy problem, domestic violence, fall from a height, fall - no injury and unspecified injury.

Other: local infection, dermatological, head injury, allergy, soft tissue inflammation, haematological, burns and scalds, urological, ophthalmological, central nervous system, ENT, muscle/tendon injury, psychiatric, dislocation/fracture/joint injury, laceration, foreign body, contusion/abrasion, poisoning (including overdose), septicaemia, bites/stings, gynaecological, sprain/ligament injury, diabetes and other endocrinological, cerebrovascular, electric shock, facio-maxillary and other vascular.

GIT category includes the following: paediatric constipation, paediatric dehydration, paediatric diarrhoea, feeding problems, paediatric infantile colic, paediatric pyloric stenosis, paediatric vomiting, Crohn's disease, gastritis, gastro-oesophageal reflux, hepatitis, inflammatory bowel disease, jaundice, liver disease, nutritional condition, neonatal feeding difficulties, neonatal failure to thrive, feeding tube problem, gallstone and swallowing problem.

Table 2 shows the original and revised diagnoses for all infants aged less than one year. A third of infants aged less than one year attended A\&E and were originally given a diagnosis of 'unclassifiable' or 'NAD'. We reduced this proportion by 42\%; 9.7\% (95\% confidence interval (Cl): $9.0,10.4)$ and 8.8\% (95\% Cl: 8.1, 9.5) for 'unclassifiable' and 'NAD', respectively.
After revision, it was felt that $14 \%$ of infants could have been given a diagnostic category code of something other than 'unclassifiable' or 'NAD' based on the provision of a clear diagnosis recorded in the medical notes. The most common reason for misclassification of the diagnostic code appeared to be that the diagnosis was written clearly in the medical notes, but not completed in the pre-assigned box for diagnosis.

Table 3 shows the presenting complaints and their frequency for those $18.5 \%$ of infants who had a confirmed diagnosis which was 'unclassifiable' or given as 'NAD'. The most common presenting complaints for infants in the 'NAD' diagnostic category was 'unsettled baby', 'diarrhoea and/or vomiting', 'abnormal breathing' and 'feeding difficulties'. The most common presenting complaints, where there was one available, for infants in the 'unclassifiable' diagnostic category, was 'fever', 'swelling or lump', 'unsettled baby' and 'diarrhoea and/or vomiting'.

Table 4 shows the diagnostic code by hospital site. The paediatric-led A\&E unit had significantly fewer (10\%) infants with a final diagnosis of 'unclassified' and significantly more infants with a diagnosis of 'NAD' or 'ID' (5\% and 8.5\%). These differences were apparent in the subsample of infants for whom the case notes were studied in more detail in prior but were less so after revision of the diagnostic code.

\section{DISCUSSION}

The majority of infants who attended A\&E were diagnosed with either ID, respiratory or gastrointestinal disorders. By detailed review of A\&E case notes, we were able to reduce the number of infants given a diagnostic category of NAD or unclassifiable by $42 \%$. Using the extrapolation of revised diagnoses in the subgroup to the whole population, we estimate that 11-12 infants per week truly attended the study A\&E services with no identifiable underlying diagnosis. There were significant differences in the proportion of infants with each diagnosis 
Table 3

\section{Presenting complaints for infants in revised categories of 'unclassifiable' and 'NAD' (\%)}

\begin{tabular}{|l|l|l|l|}
\hline Unclassifiable & & NAD & 19.0 \\
\hline Fever & 9.2 & Unsettled & 18.9 \\
\hline Lump & 7.7 & Diarrhoea and/or vomiting & 15.5 \\
\hline Unsettled & 6.2 & Feeding & 10.3 \\
\hline Follow-up & 4.6 & Cough & 6.9 \\
\hline Coryzal & 4.6 & Blood in nappy & 5.2 \\
\hline Swelling & 3.1 & Fever & 5.2 \\
\hline Fall & 3.1 & Bowels not opened & 3.4 \\
\hline Feeding & 3.1 & Odd behaviour & 3.4 \\
\hline Head injury & 3.1 & & 1.7 \\
\hline Self-discharge & 1.5 & Follow-up & \\
\hline Breathing & 1.5 & & 10.3 \\
\hline Cough & 1.5 & & \\
\hline Crying & 7.7 & & \\
\hline Diarrhoea and/or & 1.5 & & \\
\hline vomiting & 1.5 & & \\
\hline Odd behaviour & None available & \\
\hline Pain & & & \\
\hline Rash & & & \\
\hline Yellow baby & & & \\
\hline None available & & & \\
\hline NAD: no abnormality detected. & & \\
\hline
\end{tabular}

by hospital site which appeared not to reflect a true difference in the clinical diagnostic spectrum seen in each hospital. To our knowledge, this is the only published study examining the quality of A\&E attendance diagnoses for any paediatric age group. The study highlights the importance of recording a clear diagnosis for an over-represented vulnerable group of children attending
A\&E. It demonstrates the need for revision of the NHS data coding category of 'unclassifiable', as a diagnosis of unclassifiable does not necessarily mean that the diagnosis is unknown, or indeed medically insignificant. It also emphasizes the need to fully understand hospital coding systems and processes when carrying out health services research using operational data.
The attenuation of the differences in the proportion of infants in each diagnostic category after detailed review of the case notes was an interesting finding and most likely reflects underlying service rather than clinical variation. We speculate that this may have been due to factors such as the service configuration (perhaps more senior medical and nursing paediatric support in the paediatric-led unit), coding staff (same system, different staff), medical staff (more integrated in A\&E in the paediatricled unit), training of medical staff, the clerking proforma (diagnosis option clearly available at the end of the clerking form rather than on the front sheet) and/ or throughput of patients (25\% more infants seen in the A\&E led unit). It is likely that our findings can be generalized to many London DGHs and possibly to others across the United Kingdom using a similar coding system. All UK A\&E coding services use the same NHS database; however, each trust is allowed to introduce some variation in the coding system. Since these study data were collected, many A\&E services have changed their medical clerking and recording system and now use a computer-generated drop down system for diagnoses whereby the assessing doctor is either forced or has the option of choosing from a predetermined list of diagnoses before completing the healthcare episode. This list of diagnoses should reflect the NHS database for coding. In a post-hoc piece of analysis, we reviewed the diagnostic categories for infants presenting to A\&E in Hospital B for the financial year 2011-2012, as during the preceding year a new information technology (IT)-based, optional system for diagnostic recording was incorporated. The percentages of infants categorized as having NAD and unclassifiable diagnosis were $12.4 \%$ and $16.6 \%$, respectively, which is fairly similar to our revised percentages after detailed case-note review. However, a significant proportion, $31 \%$ of infants, received no diagnostic categorization at all. This suggests that there is still room for improvement in terms of ensuring doctors commit to recording 'a 
Percentage of infants in each diagnostic category by hospital site

Total sample

Audited subsample

Revised audited subsample

\section{Diagnostic category}

ID

GIT

Unclassifiable

NAD

Respiratory

Other
HA (46\%)

HB (54\%)

$p$-value

HA (45\%)

18.9

16.6

25.6

9.2

10.2

19.6

32.51

17.7

22.63

$<0.001$

8.8

19

16.87 $p$-value

HA (45\%)

HB (55\%)

$p$-value

23.3

22.22

26.2

35.4

25.2

21.8

12.2

11.5

0.045

12.54

12.5

9.5

15.8

14.4

8.2

HA: Hospital A, paediatric led (\% given refer to \% of infants of total who attended HA); HB: Hospital B - A\&E led (\% given refer to \% of infants of total who attended HA); ID: infectious disease; GIT: gastrointestinal tract (including gastroenteritis); other and unclassifiable: see Table 2; NAD: no abnormality detected.

diagnosis' from the National Data Set and thereby ensure it improves in quality over time to reflect clinical reality.

Coding errors cannot only be costly but also give an inaccurate view of hospital use, ${ }^{17}$ and therefore lead to inappropriate service configuration and provision of medical care. Furthermore, epidemiological study of the public health needs of a population will be hampered by poor data and poor understanding of data. Although we did not carry out a formal assessment of medical notes, our findings reflected poor use of standard paper proformas by medical staff, which undoubtedly led to coding errors.

Even after revised categorization, a significant proportion of infants (10\%) attended A\&E with no apparent clinical abnormality detected, suggesting that A\&E attendances may be reduced if parents could be informed on what is normal for their child at this age. An important caveat to this is that the decision to seek medical care at A\&E may be driven by other factors within the family such as mental ill-health. Mothers of young infants attending A\&E have a relatively high prevalence $(16 \%$ in Australian mothers) of postnatal depression (PND) ${ }^{18}$ and a presenting complaint of 'crying baby' has been associated with increased odds of maternal PND. ${ }^{18}$ Nursing and medical staff should therefore be alert to the possibility of factors such as PND, especially infants who do not have any apparent medical abnormality on review.

At least a quarter of infants presented with IDs, with relatively few of these infants admitted to an inpatient ward. It is likely that a proportion of these are minor self-limiting illnesses, which may be better managed in a different setting.

A number of parents and infants selfdischarged; however, this is not included in the national NHS database of diagnostic categories. Self-discharge is an important marker of the quality of a service. ${ }^{19}$

This study provides some insight into why infants aged less than one year present to A\&E; however, a more detailed review is required to answer this question in more depth, such as interview of parents and carers attending A\&E, modelling of health-care use across primary and secondary care and assessment of 'appropriateness' of A\&E attendance.

\section{CONCLUSION}

With detailed review of A\&E case notes, it was possible to reduce the proportion of infants receiving a coding diagnostic category of NAD or unclassifiable by $42 \%$. It is estimated that $10 \%$ of infants present to A\&E with no underlying medical abnormality. In our own hospitals, this would represent over 2-3 fewer infants per day who would be seen in this setting and would represent approximately $£ 47,000$ saved per year. Accurate recording and coding of diagnoses are essential to more accurately understand what parents are seeking our help for and at what cost to the economy. These findings suggest that the service configuration of a unit impacts on the quality of the coding process. This study highlights some of the pitfalls, but also the benefits of analysing routine hospital data. 
1. France N, Craze J. Medical paediatrics in the emergency department: Trends in attendance rates, sources of referral and admissions 19932005. Archives of Disease in Childhood 2006; 91(Suppl. 1): A28-30.

2. Health Social Care Information Center ( $\mathrm{HSClC}$ ). Accident and Emergency Attendances in England - 2011-12, Experimental Statistics. 23rd January 2013. Available online at: http://www. hscic.gov.uk/catalogue/PUB09624 (Last accessed 29th November 2013).

3. Child and Maternal Health Intelligence Network (ChiMat), PHE. Accident and Emergency Attendances for Children Aged 0 to 4 Years (2010-2011). Available online at: http://atlas. chimat.org.uk/IAS/dataviews/tabular?viewld=22 2\&geold=2\&subsetld = (Last accessed 29th November 2013).

4. Purdy S. Avoiding Hospital Admissions: What Does the Research Evidence Say? The King's Fund, 2010. Available online at: http://www. kingsfund.org.uk/publications/avoiding-hospitaladmissions (Last accessed 29th November 2013).

5. Saxena S, Bottle A, Gilbert R, Sharland M. Increasing short-stay unplanned hospital admissions among children in England: Time trends analysis '97-'06. PloS One 2009; 4: e7484.

6. Bedford HE, Jenkins SM, Shore C, Kenny PA. Use of an east end children's accident and emergency department for infants: A failure of primary health care? Quality in Health Care 1992; 1: 29-33.

7. Royal College of Physicians, the Royal College of General Practitioners, the Royal College of
Paediatrics and Child Health. Teams without Walls: Working Together will Improve Patient Care. 2008. Available online at: http://www.rcpch.ac.uk/news/ working-together-will-improve-patient-care-23april-2008 (Last accessed 29th November 2013).

8. Royal College of General Practitioners Child Health Strategy 2010-15: To Include Children and Young People under the Age of 19 Years. 2010. Available online at: http://www.rcgp.org.uk/clinical-andresearch/clinical-resources/ /media/Files/CIRC/ Child\%2Oand\%20Adolescent\%2OHealth/CIRC_ RCGP_Child_Health_Strategy_2010_2015_FINAL. ashx (Last accessed 29th November 2013).

9. Wahl H, Banerjee J, Manikam L, Parylo C, Lakhanpaul M. Health information needs of families attending the paediatric emergency department. Archives of Disease in Childhood 2011; 96: 335-9.

10. NHS Institute for Innovation and Improvement. Whole System Approach to Improving Emergency and Urgent Care for Children and Young People. 2011. Available online at: http://www.institute.nhs. uk/index.php?option=com_joomcart\&ltemid= 194\&main_page=document_product info\&products_id=762 (Last accessed 29th November 2013).

11. Armon K, Stephenson T, Gabriel V, MacFaul R, Eccleston P, Werneke U, Smith S. Determining the common medical presenting problems to an accident and emergency department. Arch Dis Child. 2001;84(5):390-2.

12. Jamrozik K, Samarasundera E, Miracle R, Blair M, Sethi D, Saxena S, Bowen S. Attendance for injury at accident and emergency departments in London: a cross-sectional study. Public Health. 2008;122(9):838-44.
13. Department of Education. Postcode Distances Batch Tool. Available online at: http://www. education.gov.uk/cgi-bin/inyourarea/distance.pl (Last accessed 11th October 2013).

14. Department of Education. IDACI (Income Deprivation Affecting Children Index) Batch Tool. Available online at: http://www.education.gov.uk/ cgi-bin/inyourarea/idaci.pl (Last accessed 11th October 2013).

15. Health and Social Care Information Centre: NHS Data Dictionary. Available online at: http://www. datadictionary.nhs.uk/ (Last accessed 11th October 2013).

16. Random Number Generator. Available online at: http://www.random.org (Last accessed 11th October 2013).

17. Brennan L, Watson M, Klaber R, Charles T. The importance of knowing context of hospital episode statistics when reconfiguring the NHS. British Medical Journal 2012; 344: e2432.

18. Stock A, Chin L, Babl FE, Bevan CA, Donath S, Jordan B. Postnatal depression in mothers bringing infants to the emergency department. Archives of Disease in Childhood 2013; 98: 36-40.

19. Mohsin M, Forero R, leraci S, Bauman AE, Young L, Santiano N. A population follow-up study of patients who left an emergency department without being seen by a medical officer. Emergency Medicine Journal 2007; 24: 175-9.

20. Newcombe R. Two-sided confidence intervals for the single proportion: Comparison of seven methods. Statistics in Medicine 1998; 17: 857-72.

21. Wilson EB. Probable inference, the law of succession, and statistical inference. Journal of the American Statistical Association 1927; 22: 209-12. 